\title{
FIRST RESULTS OF THE PHESAT95 CAMPAIGN \\ OF OBSERVATION OF THE PHENOMENA \\ OF THE SATELLITES OF SATURN
}

J.-E. ARLOT, W. THUILLOT, F. COLAS, D.T. VU,

J. BERTHIER, P. DESCAMPS AND CH. RUATTI

Bureau des Longitudes

Paris, France

\section{Introduction}

From 1992 to 1999, the satellites of Saturn are involved in several phenomena: eclipses or occultations by the planet, transits in front of Saturn, or transits of their umbra. These phenomena occur thanks to the geometrical circumstances in 1995 when the Earth and the Sun went through the plane of the Saturnian ring and consequently through the orbital planes of the main satellites. These circumstances are also favorable to the observation of mutual phenomena occurring when the satellites eclipse or occult each other. These mutual phenomena and the eclipses by Saturn are rare as they occur only every 15 years. But, they are very useful to get highly accurate astrometric observations and they allowed us to estimate some physical parameters. We have organized the PHESAT95 campaign to observe these phenomena and a special effort was made to observe as many events as possible. At the present time, the observations are still being collected from different teams and different countries; this paper shows the first results which have been obtained in several sites, and the first comparison with the theory of the motions.

\section{The Phenomena}

Every 15 years, the Earth and the Sun cross the equatorial plane of Saturn. Fortunately, the 1995 opposition of Saturn was close to the crossing plane event: the 1995 period of phenomena was a favorable one. During about three years, from 1994 to 1996 several eclipses by the planet and mutual events were observable. Predictions of these phenomena have been made (Arlot and Thuillot, 1993; Aksnes and Dourneau, 1994), and have shown

\section{M. Wytrzyszczak, J. H. Lieske and R. A. Feldman (eds.),}

Dynamics and Astrometry of Natural and Artificial Celestial Bodies, 531, 1997.

(C) 1997 Kluwer Academic Publishers. Printed in the Netherlands. 
TABLE 1. Main sites of the PHESAT95 network with the number of mutual events (Mut.) and eclipses by Saturn (Ecl.) observed; $\left({ }^{*}\right)$ : not yet available.

\begin{tabular}{lccllccc}
\hline Location & Country & Mut. & Ecl. Location & Country & Mut. Ecl. \\
\hline Assy Obs. & Kazakhstan & 2 & Itajuba Obs. & Brazil & $\left(^{*}\right)$ & \\
Bangalore Obs. & India & $\left(^{*}\right)$ & Lumezzane & Italy & 3 & \\
Bordeaux Obs. & France & $12\left(^{*}\right)$ & Meudon Obs. & France & 7 & \\
Bucarest Obs. & Romania & 1 & OHP (St Michel) & France & 16 & 2 \\
Catania Obs. & Italy & 10 & Pic du Midi & France & 10 & 16 \\
Charlottesville & USA & 4 & Saint Véran & France & 3 & \\
Chelmsford & U.K. & 2 & & Sternberg lab. & Krymea & 3 & \\
ESO & Chile & 20 & 2 & Stuttgart & Germany & 1 \\
\hline
\end{tabular}

that 159 eclipses and 182 mutual events at more than 5 arcsec from the edge of Saturn were observable (Thuillot and Arlot, 1995).

These geometric circumstances are in fact favorable to observe several phenomena by Saturn: eclipses, occultations, transits and transits of umbra. Among them, eclipses are the most convenient to observe with photometric detectors, but the proximity of Saturn and its rings is a real difficulty which assigns these observations mainly to CCD detectors installed in good sites. On the other hand, mutual events may occur much farther from the planet. Furthermore, the mutual eclipses and occultations do not involve any atmosphere (except when Titan is implied) and thus, their astrometric analysis can lead to a better accuracy.

\section{The PHESAT95 Campaign of Observation}

At the Bureau des longitudes, we have organized an international campaign to observe the phenomena of the Saturnian satellites. The goal of this campaign was to get high accuracy astrometric measurements of these satellites thanks to photometric observations with CCD cameras or photometers. These observations will be used to improve the dynamical models of their motions.

In order to collect the maximum of observations, sites with various longitudes were included in the PHESAT95 network. Table 1 gives the names and countries of the main sites of this network. CCD cameras have generally been used; only a few observers used photometers which are more difficult to use because of the brightness of the planet.

\section{Preliminary Analysis of the Observations}

Observational data are still being collected. We can have a general status of the results obtained: mainly mutual events have been observed. At the 
TABLE 2. Number of eclipses disappearances (ECD) and reappearances (ECR) by satellites.

\begin{tabular}{lccccccc}
\hline Sat. & 1 & 2 & 3 & 4 & 5 & 6 & 7 \\
\hline ECD & - & - & 1 & 1 & 2 & 1 & - \\
ECR & 2 & 3 & 5 & 2 & 3 & - & - \\
\hline
\end{tabular}

TABLE 3. Number of mutual events (A eclipses/occults B) by satellites for the 57 eclipses and 27 occultations collected.

\begin{tabular}{ccccccccc}
\hline Sat. B & & 1 & 2 & 3 & 4 & 5 & 6 & 7 \\
\hline Sat. A & 1 & - & - & - & - & - & - & - \\
& 2 & $1 / 3$ & - & $2 / 1$ & $-/ 2$ & - & - & - \\
& 3 & $15 /-$ & $5 / 5$ & - & $-/ 2$ & $5 /-$ & $1 /-$ & $1 /-$ \\
& 4 & - & $3 / 3$ & $3 / 4$ & - & $7 / 1$ & $-/ 2$ & - \\
& 5 & $2 /-$ & $2 /-$ & $1 /-$ & $3 / 4$ & - & $2 /-$ & - \\
& 6 & $2 /-$ & $2 /-$ & - & - & - & - & - \\
& 7 & - & - & - & - & - & - & - \\
\hline
\end{tabular}

present time, as shown in Table 1, we get 94 mutual events observations and 20 eclipses by Saturn; all these observations are raw data and they must now be analyzed. Models of lightcurves, similar to those we used for the mutual events of the Galilean satellites (Descamps et al., 1992; Descamps, 1994), have to be applied in order to deduce the best accurate astrometric data and some physical parameters related to the surface parameters.

The available observations, regarding the involved satellites, are in $\mathrm{Ta}$ ble 2 for the eclipses by Saturn, and in Table 3 for the mutual eclipses and mutual occultations. Contrarily to positional observations, the observations of events do not give uniform sets of data: events implying fast satellites (but not too close to the planet) are more numerous and more often observed than events involving distant and slow moving satellites. In these tables Hyperion is involved in only one phenomenon. But, this type of observation allows us to get more accurate measurements. Table 4 shows the preliminary residuals obtained for some observations made at the Pic du Midi, Meudon and Haute-Provence observatories. Residuals are computed thanks to Dourneau's theory of the motions (Dourneau, 1993). All of these observations have been done with THX7863 CCD cameras.

\section{Conclusion}

For the observations of the Saturnian satellites, mutual events and eclipses are a rare opportunity to get accurate astrometric measurements. We 
TABLE 4. Preliminary residuals obtained for observations made at the Pic du Midi (PDM), Meudon observatory (MEU) and Observatoire de Haute-Provence (OHP) in France.

\begin{tabular}{lcrll}
\hline $\begin{array}{l}\text { Date } \\
1995\end{array}$ & Event & $\begin{array}{r}\text { C-O } \\
{[\mathrm{s}]}\end{array}$ & $\begin{array}{c}\text { Prec. } \\
{[\mathrm{s}]}\end{array}$ & Observatory \\
\hline June 17 & $2 \mathrm{E} 3$ & 3 & \pm 15 & OHP \\
July 22 & $3 \mathrm{E} 2$ & 15 & \pm 10 & OHP \\
Aug. 25 & $3 \mathrm{O} 2$ & 14 & \pm 3 & OHP \\
Sept. 21 & $4 \mathrm{O} 3$ & 21 & \pm 21 & PDM \\
Sept. 24 & $3 \mathrm{E} 5$ & 17 & \pm 17 & OHP \\
Nov. 9 & $6 \mathrm{E} 2$ & -68 & \pm 6 & PDM \\
Nov. 14 & $4 \mathrm{E} 2$ & 7 & \pm 2 & OHP \\
Nov. 14 & $5 \mathrm{E} 2$ & 24 & \pm 18 & OHP \\
Nov. 18 & $5 \mathrm{E} 6$ & 16 & \pm 55 & MEU \\
Nov. 18 & $5 \mathrm{E} 4$ & -13 & \pm 4 & MEU \\
Nov. 27 & $5 \mathrm{E} 2$ & 0 & \pm 49 & MEU \\
\hline
\end{tabular}

are now at the end of the PHESAT95 campaign that we organized in order to catch the maximum of these rare events. The first results, we obtained, show the good quality of the observations made. We have now to collect the whole set of observations, to apply homogeneous methods of analysis and reduction, and then to apply this material to the improvement of the dynamical model of the motions. Global results will lead the observers to publish a catalogue as it has been done during the previous campaign involving the Jovian satellites (PHEMU91 observers, 1996). On the other hand, we have also prepared the new campaign of observations of the mutual events of the Galilean satellites (Arlot, 1996; Arlot et al., 1996) which will occur during the next year. Further information on these campaigns are available on the WEB server of the Bureau des longitudes (http://www.bdl.fr).

\section{References}

Aksnes, K. and Dourneau, G.: 1994, Icarus 112, 545.

Arlot, J.-E., Descamps, P., and Thuillot, W.: 1996, Icarus, to be published.

Arlot, J.-E. and Thuillot, W.: 1993, Icarus 105, 427.

Arlot, J.-E.: 1996, Astron. Astrophys., to be published.

Descamps, P., Arlot, J.-E., Thuillot, W., Colas, F., Vu, D.T., Bouchet, P., and Hainaut, O.: 1992, Icarus 100, 235.

Descamps, P.: 1994, Astron. Astrophys. 291, 664.

Dourneau, G.: 1993, Astron. Astrophys. 267, 292.

PHEMU91 observers: 1996, Astron. Astrophys., to be published.

Thuillot, W. and Arlot, J.-E.: 1996, "Sur l'observation de phénomènes des satellites de Saturne", Suppl. Ann. Physique 21, 1-192, (also available on the Web Server http://www.bdl.fr/PHESAT95/phesat95.html) 